\title{
IRF2-INPP4B-mediated autophagy suppresses apoptosis in acute myeloid leukemia cells
}

\author{
Feng Zhang ${ }^{1}$, Jiajia Li ${ }^{1}$, Junfeng Zhu' ${ }^{1}$ Lin Liu ${ }^{1}$, Kai Zhu' ${ }^{1}$ Shuang Cheng ${ }^{2}$, RuDi Lv ${ }^{3}$ and Pingping Zhang ${ }^{\text {** }}$
}

\begin{abstract}
Background: The present study aimed to investigate the underlying role of interferon-regulatory factor 2 (IRF2)-inositol polyphosphate-4-phosphatase, type-II (INPP4B) axis in the regulation of autophagy in acute myeloid leukemia (AML) cells.

Methods: Quantitative real time PCR (QRT-PCR) and western blot were performed to determine the expression levels of IRF2, INPP4B and autophagy-related markers in AML cell lines. Autophagy was assessed by elevated Beclin-1 expression, the conversion of light chain 3 (LC3)-I to LC3-II, downregulated p62 expression and green fluorescent protein (GFP)-LC3 puncta formation. The colony formation and apoptosis assays were performed to determine the effects of IRF2 and INPP4B on the growth of AML cells.
\end{abstract}

Results: IRF2 and INPP4B were highly expressed in AML cell lines, and were positively correlated with autophagyrelated proteins. Overexpression of IRF2 or INPP4B stimulated autophagy of AML cells, whereas inhibition of IRF2 or INPP4B resulted in the attenuation of autophagy. More importantly, IRF2 or INPP4B overexpression reversed autophagy inhibitor, 3-methyladenine (3-MA)-induced proliferation-inhibitory and pro-apoptotic effects, while IRF2 or INPP4B silencing overturned the proliferation-promoting and anti-apoptotic effects of autophagy activator rapamycin.

Conclusion: IRF2-INPP4B signaling axis attenuated apoptosis through induction of autophagy in AML cells.

Keywords: IRF2, INPP4B, Autophagy, Apoptosis, Acute myeloid leukemia

\section{Background}

Acute myeloid leukemia (AML) is a hematopoietic malignancy characterized by the abnormal proliferation of undifferentiated myeloid precursors and impaired hematopoiesis [1]. As the most common type of acute leukemia in adults, AML develops rapidly, resulting in a low long-term survival rate, and its incidence increases with increasing age. Although $50-75 \%$ patients with AML have a response to chemotherapy, relapse represents the major cause of treatment failure [2]. So far, the pathogenesis of AML has not been fully elucidated.

*Correspondence: 704078480@qq.com

1 Department of Hematology, The First Affiliated Hospital of Bengbu Medical College, No. 287 Changhuai Road, Bengbu 233004, Anhui, People's Republic of China

Full list of author information is available at the end of the article
It has become increasingly clear that autophagy might be involved in a variety of multiple biological processes (e.g., cell survival, aging and death) and implicated in metabolic diseases, tumors, degenerative diseases, aging and infection [3, 4]. Several lines of evidence suggest that autophagy and apoptosis share a signaling-dependent regulated process that allows the degradation of some cellular proteins in autophagosomes essential for maintaining cell homeostasis and organelle renewal [5]. At present, autophagy is generally regarded as a regulatory mechanism of defense and a survival response to stress [6]. Apoptosis, known as a form of programmed cell death, is a critical component in discarding unsalvageable cells or inhibiting overgrowth. Autophagy, on the other hand, initially attempts to save the injured cells. However, autophagy behaves oppositely and cooperates with apoptosis following metabolic stress subsequently 
accelerates cell death $[7,8]$. Therefore, the imbalance between autophagy and apoptosis potentially leads to tumorigenesis. Liu and colleagues reported that autophagy-related gene 5 (Atg5)-dependent autophagy contributed to AML development [9]. Watson et al. demonstrated that loss of Atg5 resulted in an identical hematopoietic stem and progenitor population (HSPC) phenotype as loss of Atg7, confirming a general role for autophagy in HSPC regulation [10]. Moreover, Folkerts et al. showed that knockdown of Atg5 inhibited myeloid leukemia maintenance [11], indicating that targeting autophagy might provide new therapeutic options for treatment of AML.

The interferon regulatory factor (IRF) proteins family are the crucial factors in immunoregulation, cell proliferation regulation, hematopoietic stem cell development, lymphocyte differentiation and cellular response that is involved in tumorigenesis [12]. The IRF2 gene, a member of IRF family, is located on chromosome 4. Our previous study [13] demonstrated that shRNA-mediated IRF2 knockdown suppressed cell growth and colony formation, down-regulated the level of anti-apoptotic factor Bcl-2 and up-regulated the protein levels of apoptotic proteins Bax and the cleaved caspase 3 in AML cell lines OCI/ AML-2, OCI/AML-3, and THP-1 cells. Further investigation showed that IRF2 upregulated inositol polyphosphate-4-phosphatase, type-II (INPP4B) expression via binding to INPP4B promoter, which in turn inhibited cell apoptosis in AML cells. Nevertheless, the detailed mechanism by which INPP4B inhibited AML cell apoptosis remained unclear. As was mentioned above, we hypothesis that IRF2 might regulate cell autophagy through interacting with INPP4B, thereby affecting the growth and apoptosis of AML cells, and ultimately participating in the induction of AML development.

\section{Materials and methods \\ Cell lines}

AML cell lines (OCI/AML-2, OCI/AML-3, Kasumi-3, PL-21, MV-4-11, CESS, Kasumi-1, BDCM and THP1) purchased from American Type Culture Collection (ATCC, Manassas, VA, USA) were maintained in $\alpha$-minimal essential medium (MEM) supplemented with $10 \%$ fetal bovine serum (FBS), $100 \mathrm{U} / \mathrm{mL}$ penicillin and $100 \mu \mathrm{g} / \mathrm{mL}$ streptomycin (all from Invitrogen, Carlsbad, CA, USA) at $37^{\circ} \mathrm{C}$ in humidified $5 \% \mathrm{CO}_{2}$ and $95 \%$ air.

\section{Transient transfections and treatments}

Full-length IRF2 and INPP4B were amplified and cloned into the pcDNA3.1 expression vector which was then stably transfected into OCI/AML-2 or THP-1 cells for IRF2 and INPP4B overexpression, while small interference RNA (siRNA) targeting IRF2 (si-IRF2) and INPP4B
(si-INPP4B) oligos and control siRNA (si-Ctrl) were used to construct the knockdown models and negative controls, which were all designed and synthesized by Shanghai GenePharma Co., Ltd. (Shanghai, China). The qRT-PCR and western blotting were used to detect the transfection efficiency. Additionally, the protein levels of autophagy-related genes Beclin-1, microtubuleassociated protein light chain 3 (LC3)-I, LC3-II and p62 were also examined. The green fluorescent protein (GFP)-LC3 puncta formation was also evaluated by immunofluorescence.

OCI/AML-2 and THP-1 cells were transfected with IRF2, INPP4B, si-IRF2, si-INPP4B or their negative controls for $48 \mathrm{~h}$ using Lipofectamine 2000 (Invitrogen) after treatment with autophagy inhibitor 3-methyladenine (3-MA; $10 \mathrm{mmol} / \mathrm{L}$; Sigma-Aldrich, St. Louis, MO, USA), autophagy activator rapamycin $(10 \mu \mathrm{g} / \mathrm{L}$; LC Laboratories, Woburn, MA, USA) or $0.1 \%$ dimethylsulfoxide (DMSO; Sigma-Aldrich) for another $24 \mathrm{~h}$. Cells were collected for determining the expression of autophagyrelated proteins, autophagosome accumulation, proliferation and apoptosis.

\section{Immunofluorescence}

OCI/AML-2 or THP-1 cells following transfection with IRF2, INPP4B, si-IRF2, si-INPP4B or their negative controls were seeded into a $35 \mathrm{~mm}$ diameter petri dish covered with a glass slide (microscope cover glass, $18 \times 18 \mathrm{~mm}$ ) at a density of $8 \times 10^{4}$ cells $/ \mathrm{mL}$ and maintained at $37{ }^{\circ} \mathrm{C}$ with $5 \% \mathrm{CO}_{2}$ for $24 \mathrm{~h}$. These cells were transiently transfected with GFP-LC3 plasmid using Lipofectamine 2000 (Invitrogen) according to the manufacturer's specification. After $48 \mathrm{~h}$ of posttransfection, the slides were rinsed with PBS for three times, fixed in $4 \%$ paraformaldehyde for $10 \mathrm{~min}$, washed again with PBS for three times, and sealed with anti-fluorescence quenching mounting medium $(15 \mu \mathrm{L})$. Fluorescence levels of GFPLC3 were detected by using laser confocal microscopy (CLSM; Leica, Wetzlar, Germany; magnification $60 \times$ ). To quantify autophagic cells, we counted the number of autophagic cells demonstrating GFP-LC3 dots $(\geq 10$ dots/cell) among 200 GFP-positive cells.

\section{RNA extraction and PCR analysis}

Total RNA was extracted from AML cell lines using Trizol reagent (Invitrogen), and reversely transcribed into cDNA using the SuperScript III kit (Invitrogen) according to the manufacturer's protocol. The relative quantification of IRF2, INPP4B, Beclin-1 and LC3 mRNA expression levels were determined using Real-Time Quantitative PCR SYBR Green kit (Takara, Tokyo, Japan) on an ABI 7500 Real-Time PCR system (Applied Biosystems, Carlsbad, 
CA, USA) and calculated by the $2^{-\Delta \Delta C t}$ method. GAPDH served as an internal control.

\section{Western blot}

Total protein was separated from AML cell lines using Radio-Immunoprecipitation Assay (RIPA) buffer (Santa Cruz Biotechnology, Santa Cruz, CA, USA), incubated with $6 \%$ sodium dodecyl sulfate polyacrylamide gel electrophoresis (SDS-PAGE; Beyotime, Shanghai, China) and transferred to polyvinylidene fluoride (PVDF) membranes (Bio-Rad, Hercules, CA, USA). Afterwards, the membranes were blocked with tris buffered saline tween (TBST; $1.5 \mathrm{mM}$ Tris, $5 \mathrm{mM} \mathrm{NaCl}, 0.1 \%$ Tween20) containing $5 \%$ skim milk at room temperature for $1 \mathrm{~h}$ and probed with primary antibodies against IRF2, INPP4B, Beclin-1, LC3-I, LC3-II and p62 (1:1000 dilution; all from Cell Signaling Technology, Boston, MA, USA) at $4{ }^{\circ} \mathrm{C}$ overnight. The secondary antibody was horseradish peroxidase-labeled antibody (1:5000), and $\beta$-actin was used as an internal control. Band intensities were standardized and the relative density was analyzed on a Molecular Imager ChemiDoc XRS System (Bio-Rad Laboratories, Hercules, CA, USA) using enhanced chemiluminescence reagent (Thermo Scientific, Shanghai, China).

\section{Cell proliferation assay}

The proliferation of OCI/AML-2 and THP-1 cells was assessed by colony formation assay. Approximately 5000 cells were seeded in $35 \mathrm{~mm}$ plates and incubated at $37{ }^{\circ} \mathrm{C}$ for 9 days. Subsequently, these cells were washed twice with PBS, and stained with $0.1 \%$ crystal violet (Beyotime, Shanghai, China) for $15 \mathrm{~min}$ at room temperature. The number of colonies was counted under an inverted microscope (Leica).

\section{Cell apoptosis assay}

Cell apoptosis was analyzed by flow cytometry using Annexin V-FITC apoptosis detection kit (BD Biosciences; San Jose, CA, USA) according to the manufacturer's protocols. OCI/AML-2 and THP-1 cells following transfection or different treatments were collected, washed with cold PBS and stained with binding buffer containing Annexin V-FITC and propidium iodide (PI) at $4{ }^{\circ} \mathrm{C}$ under darkness for $15 \mathrm{~min}$. Finally, cells were recorded using flow cytometry (Beckman Coulter, Fullerton, CA, USA).

\section{Statistical analysis}

Data were presented as mean \pm standard deviation (SD) and analyzed using SPSS 22.0 (IBM, Armonk, NY, USA). Two or more data sets were compared using Student's t-test or one-way analysis of variance, with $\mathrm{P}<0.05$ being considered statistically significant. Correlation analysis was performed using the Spearman's rank test.

\section{Results}

IRF2 and INPP4B were positively correlated with autophagy-related genes in AML cells We firstly analyzed the mRNA expression patterns of IRF2, INPP4B, Beclin-1 and LC3 in 9 AML cell lines. The results indicated that the mRNA expression levels of IRF2 and INPP4B were similar to that of Beclin-1 and LC3 (Fig. 1a). In addition, a positive correlation was observed between the mRNA expression of IRF2 or INPP4B and Beclin-1 or LC3 (Fig. 1b). Taken together, our data suggested that IRF2 and INPP4B might be involved in the autophagy of AML cells.

\section{IRF2-INPP4B axis promoted autophagy in AML cells}

We next performed in vitro gain- and loss-of-function experiments in AML cells to investigate whether IRF2 and INPP4B could promote the autophagy of AML cells. Satisfactory transfection efficiency was obtained after $48 \mathrm{~h}$ transfection with IRF2 or INPP4B expression vector and siRNA targeting IRF2 or INPP4B plasmids as determined by qRT-PCR (Fig. 2a) and western blotting (Fig. 2b).

Furthermore, overexpression of IRF2 and INPP4B in OCI/AML-2 and THP-1 cells resulted in enhanced conversion of LC3-I into LC3-II, p62 degradation, high levels of Beclin-1 (Fig. 2c) and increased GFP-LC3-positive cells (Fig. 2d). In contrast, siRNA-mediated silencing of IRF2 and INPP4B decreased Beclin-1 protein level and LC3-II/ LC3-I ratio, upregulated p62 (Fig. 3a) and reduced LC3 dots formation (Fig. 3b). Besides, INPP43 knockdown overturned the effect of IRF2 overexpression on the protein expression of autophagy-related markers (Fig. 4a) and the formation of LC3 positive puncta (Fig. 4b). Collectively, these results indicated that IRF2-INPP4B axis triggered the autophagy of AML cells.

\section{IRF2-INPP4B-induced autophagy inhibited apoptosis in AML cells}

To further determine whether IRF2-INPP4B axis alleviated cell apoptosis through activation of autophagy, we used autophagy inhibitor, 3-MA to block autophagy process. Colony formation assay revealed that 3-MA suppressed and IRF2 or INPP4B overexpression promoted cell proliferation of AML cells, while enforced expression of IRF2 or INPP4B negated the inhibitory effect of 3-MA on the proliferation (Fig. 5a). Moreover, results from flow cytometry demonstrated that 3-MA promoted but IRF2 and INPP4B overexpression suppressed AML cell apoptosis (Fig. 5b). We also turned to autophagy inducer rapamycin treatment to further establish a functional importance of IRF2 and INPP4B knockdown in autophagy-related cellular phenotypes. Mechanistic 
a
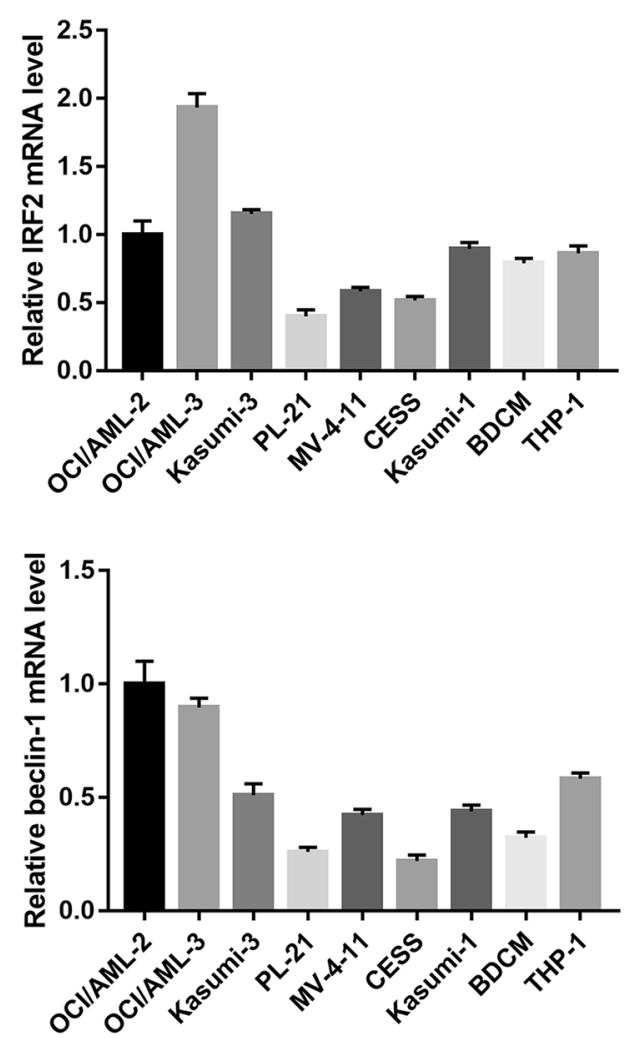

b
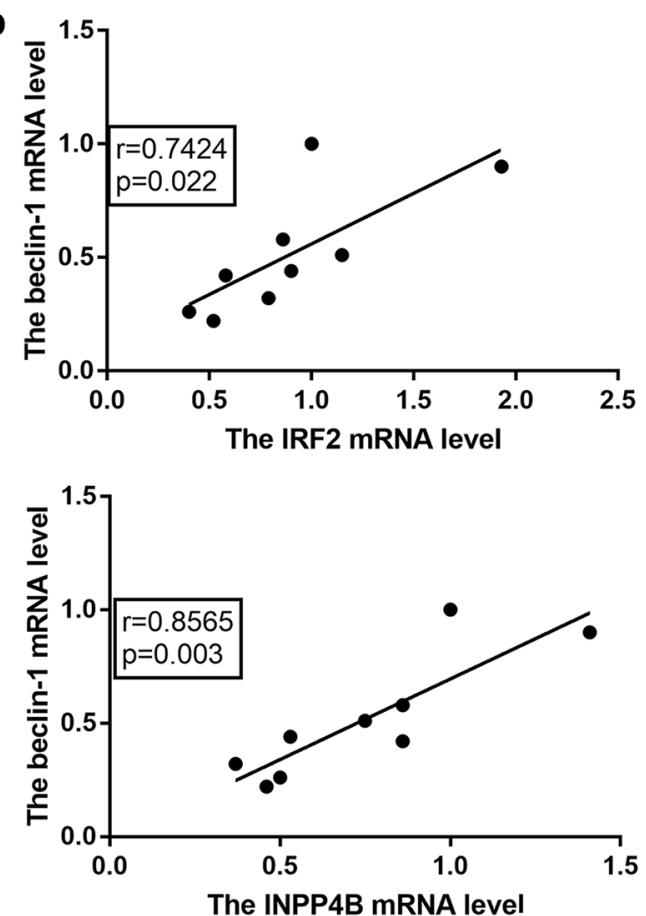
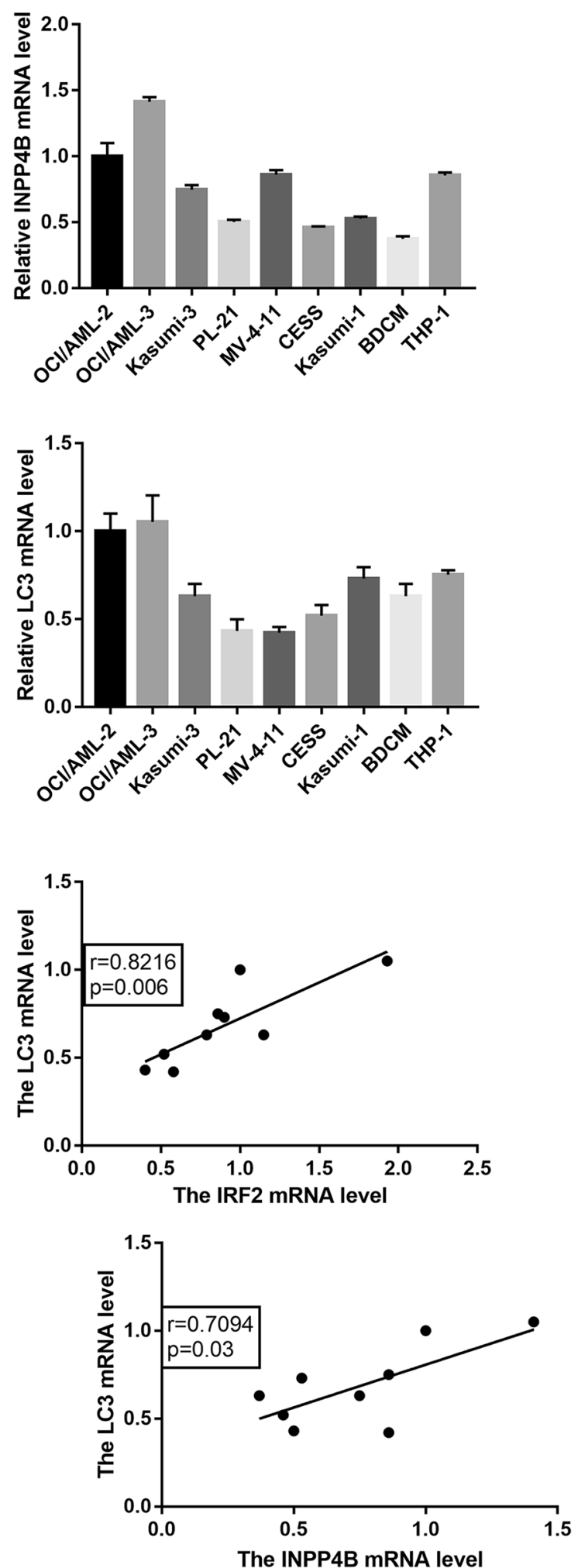

Fig. 1 The expression and correlation of IRF2, INPP4B and autophagy-related genes in AML cell lines. The mRNA expression levels (a) and correlation analysis (b) of IRF2, INPP4B, Beclin-1 and LC3 in 9 AML cell lines OCI/AML-2, OCI/AML-3, Kasumi-3, PL-21, MV-4-11, CESS, Kasumi-1, BDCM and THP-1 cells 


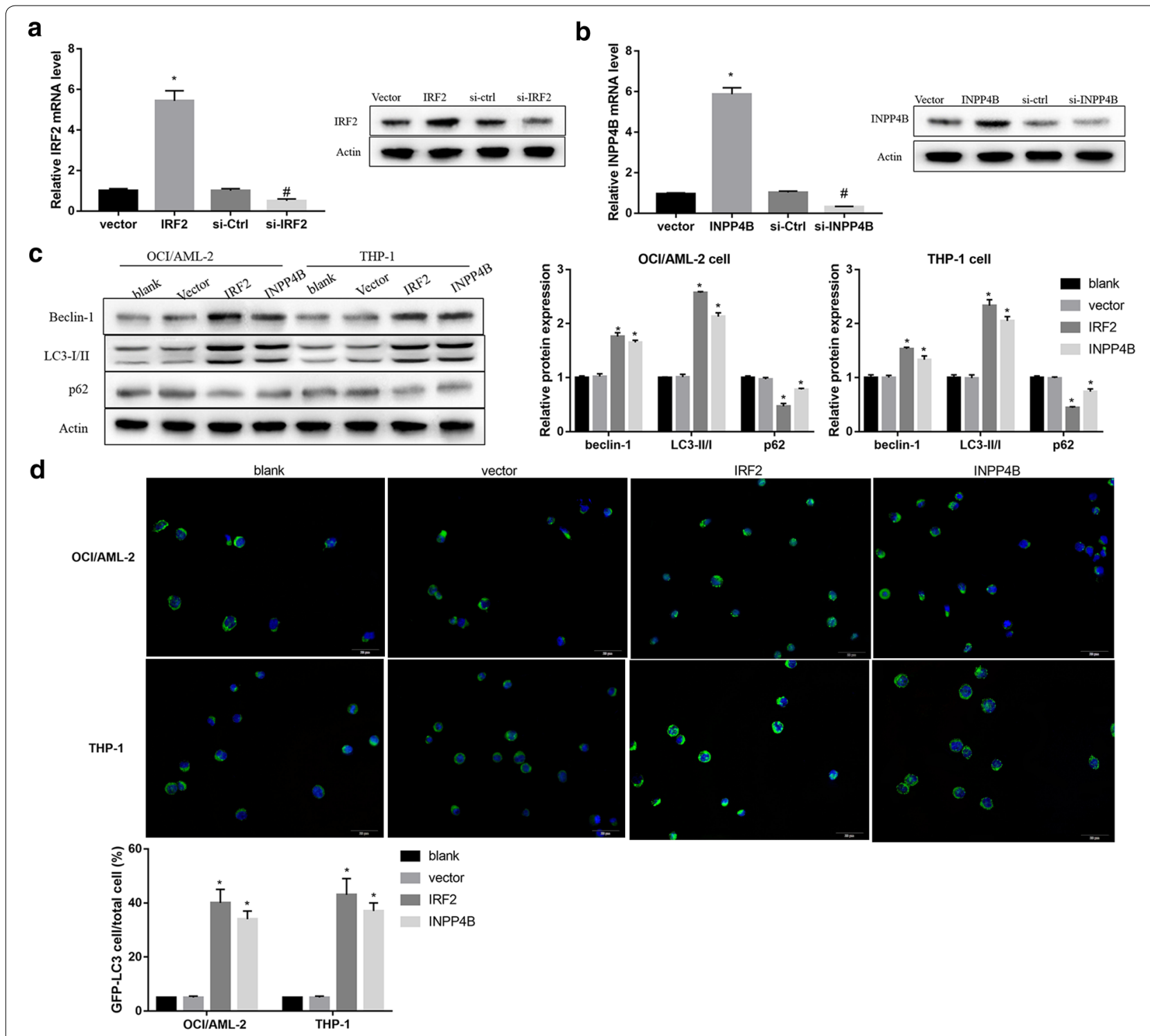

Fig. 2 The effect of IRF2 or INPP4B overexpression on the autophagy of AML cells. a The mRNA and protein levels of IRF2 in OCI/AML-2 cells transfected with vector, IRF2, si-Ctrl and si-IRF2. b The mRNA and protein levels of INPP4B in OCI/AML-2 cells transfected with vector, INPP4B, si-Ctrl and si-INPP4B. The protein levels of Beclin-1, LC3-I, LC3-II (c) and P62 and the labeled number of GFP-LC3 vacuoles-positive cells (d) in OCI/AML-2 and THP-1 cells in the groups of blank, vector, IRF 2 and INPP4B. ${ }^{*} P<0.05$ vs. the vector-transfected cells; ${ }^{*} \mathrm{P}<0.05$ vs. the si-Ctrl transfected cells

analyses further revealed that rapamycin markedly facilitated the proliferation of AML cells (Fig. 5c) and suppressed apoptosis (Fig. 5d), which were reversed by IRF2 or INPP4B knockdown. Jointly, our findings manifested that IRF2-INPP4B axis inhibited apoptosis via inducing autophagy in AML cells.

\section{Discussion}

To date, the role of autophagy in AML development has been poorly investigated. In this work, we first attempted to understand the potential mechanism that IRF2 regulated cellular apoptosis and autophagy in AML cell lines, and finally proved that IRF2 induced autophagy and inhibited apoptosis in AML cells through binding to INPP4B promoter.

In various kinds of cancers, IRF2 showed its tumorsuppressive roles, or oncogenic functions. For instance, IRF2 expression detected by immunohistochemistry was significantly downregulated in gastric cancer (GC) tissues compared to the nontumor tissues [14]. Yi et al. [15] found that hepatocellular carcinoma (HCC) patients with high IRF2 expression had lower serum alpha-fetoprotein 


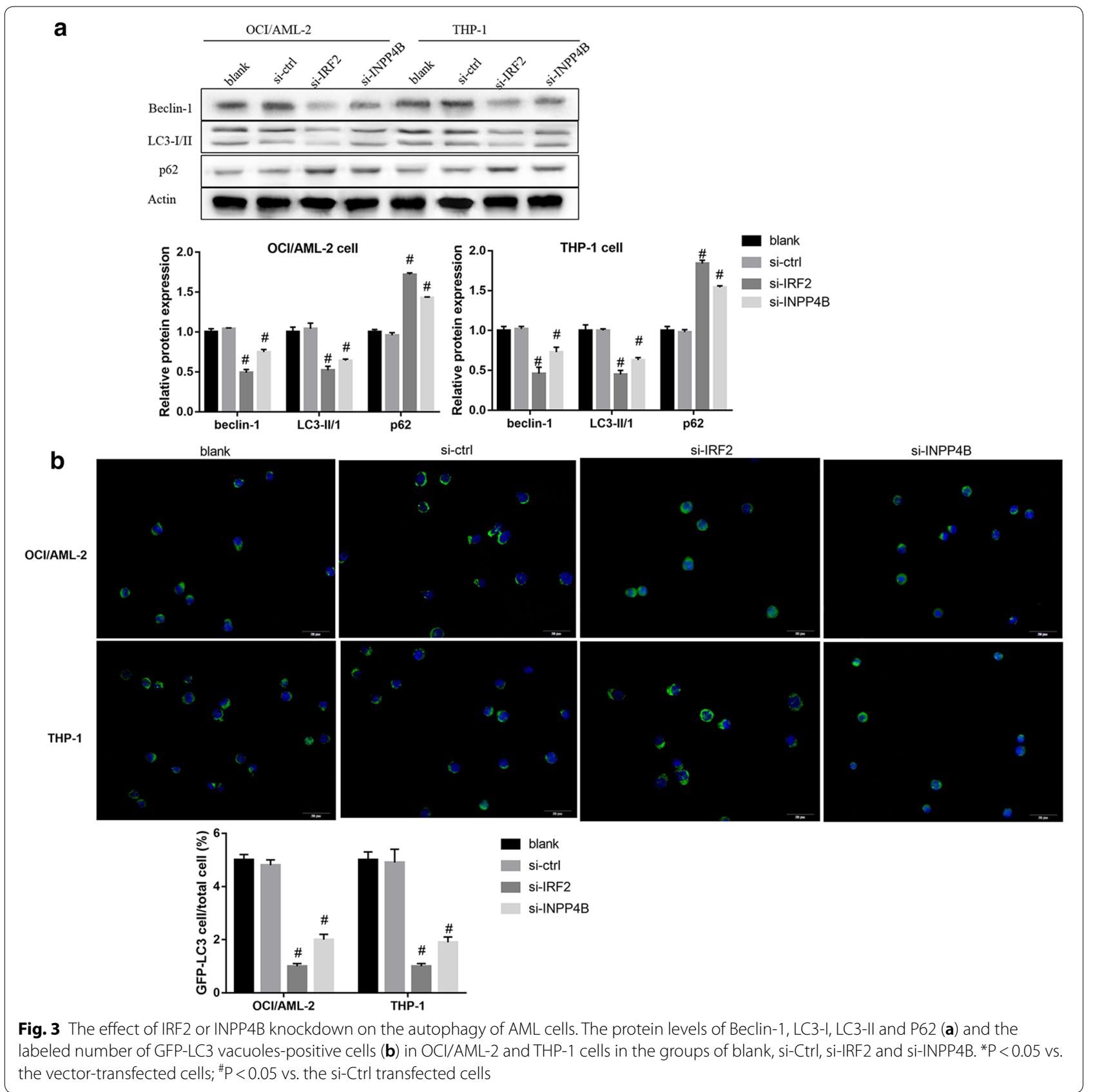

(AFP) levels, tumor differentiation, and vascular invasion and tumor-node-metastasis (TNM) stage. On the contrary, Sakai et al. [16] demonstrated that IRF2 protein levels were observably increased in human pancreatic cancer specimens as compared with the paired normal areas of the pancreas and were associated with worse features of tumor infiltration depth and overall survival (OS). The higher expression of IRF2 were observed in colorectal cancer (CRC) tissues compared to those in paired normal tissues and were significantly associated with distant metastasis and worse OS as well as TNM stage, indicating that IRF2 functioned as an independent prognostic factor in CRC [17]. Our present study suggested that IRF2 expression was significantly upregulated in AML cell lines. More importantly, the expression of IRF2 was positively correlated with the mRNA expression of autophagy-related proteins, revealing that IRF2 might be involved in autophagy of AML cells.

Besides, IRF2 is reported to influence the occurrence and development of some cancers through proliferation, 

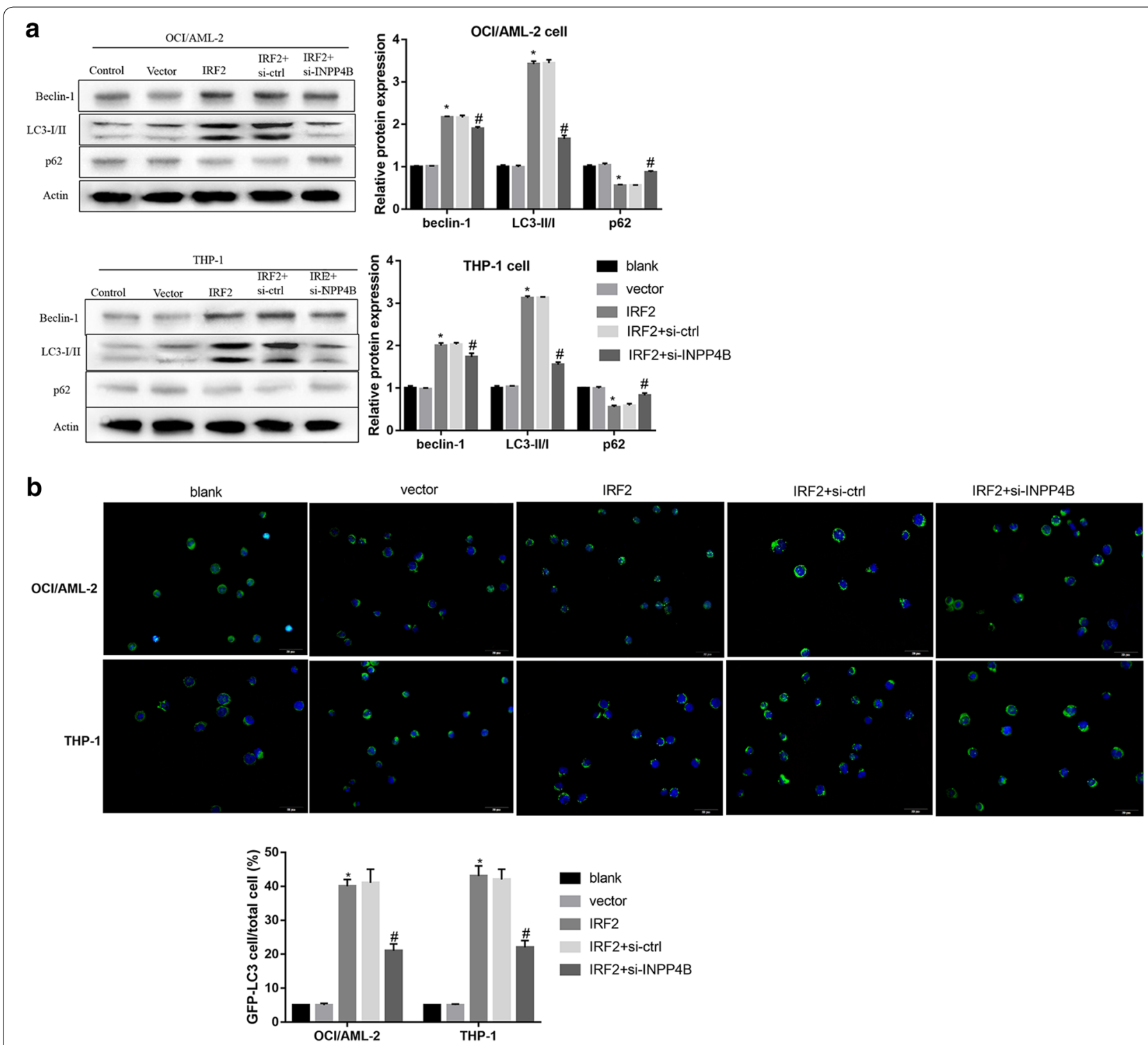

Fig. 4 The possible mechanism of IRF2-INPP4B-induced apoptosis inhibition. The protein levels of Beclin-1, LC3-I, LC3-II and P62 (a) and the labeled number of GFP-LC3 vacuoles-positive cells (b) in OCI/AML-2 and THP-1 cells in the groups of control, vector, IRF2, IRF2 + si-Ctrl and IRF2 + si-INPP4B. ${ }^{*} P<0.05$ vs. the vector-transfected cells; ${ }^{\# P}<0.05$ vs. the IRF $2+$ si-Ctrl transfected cells

apoptosis and metastasis via altering its target genes. For example, Choo et al. [18] showed that IRF2 knockdown exhibited the obviously decreased proliferation and cell cycle and induction of polyploidy, differentiation and apoptosis in leukaemic cells. Additionally, downregulation of IRF2 significantly decreased cell proliferation of testicular embryonal carcinoma (NT2) cells by elevating p53 expression [19]. Overexpression of IRF2 can significantly inhibit the non small cell lung cancer (NSCLC) cell proliferation and invasion [20]. In addition, forced expression of IRF2 increased the expressions of proliferation-related genes cyclin D1 and proliferating cell nuclear antigen (PCNA), suggesting that IRF2 exerted oncogenic activities in human pancreatic cancer [16]. Our previous study provided the evidence that IRF2 was an important regulator of AML cell growth, colony formation and survival [13]. In accordance with previous reports, our findings revealed that IRF2 overexpression promoted cell autophagy and proliferation and inhibited cell apoptosis in AML cell lines OCI/AML-2 and THP-1, whereas IRF2 silencing led to an opposite effect, suggesting that IRF2 plays a crucial role in AML progression via autophagy induction. 
a

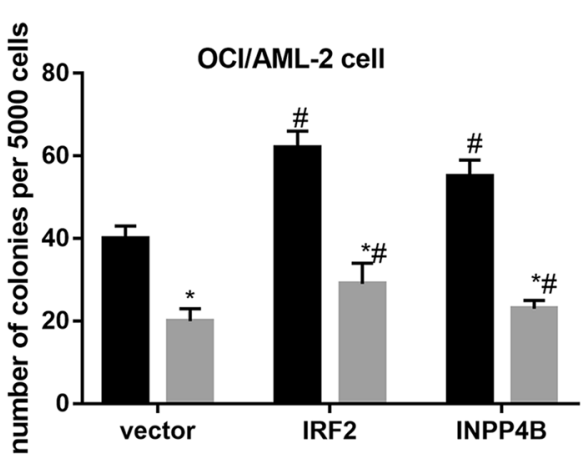

b

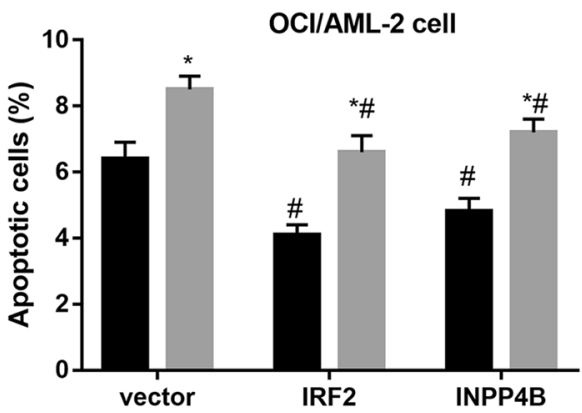

C

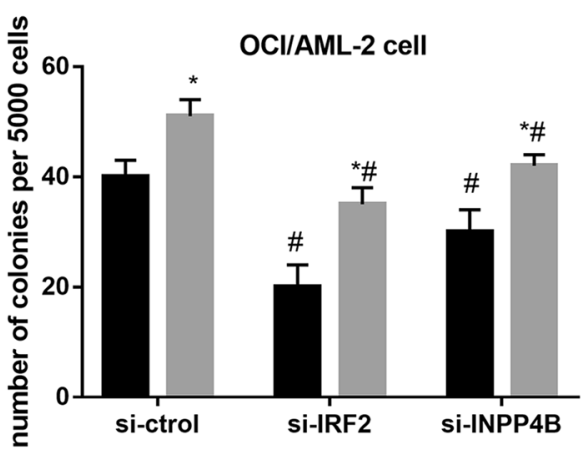

d

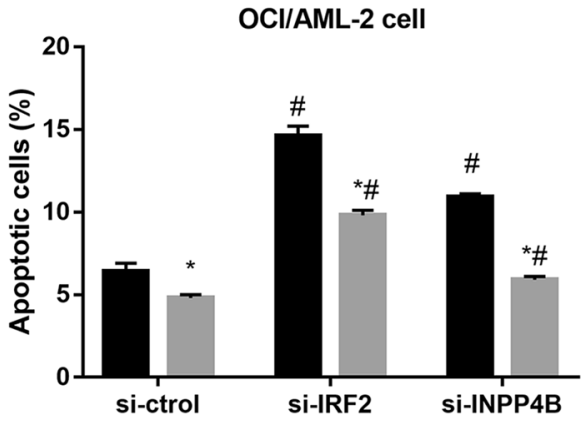

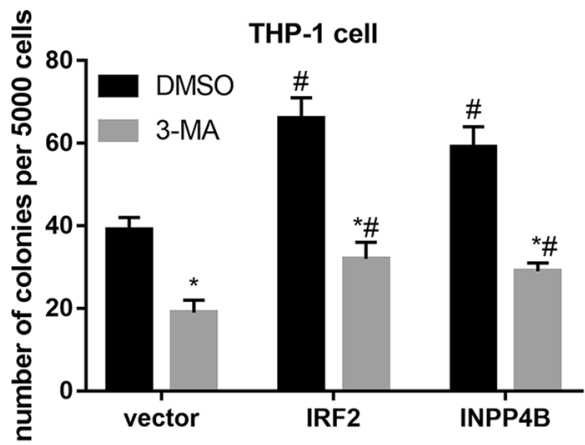
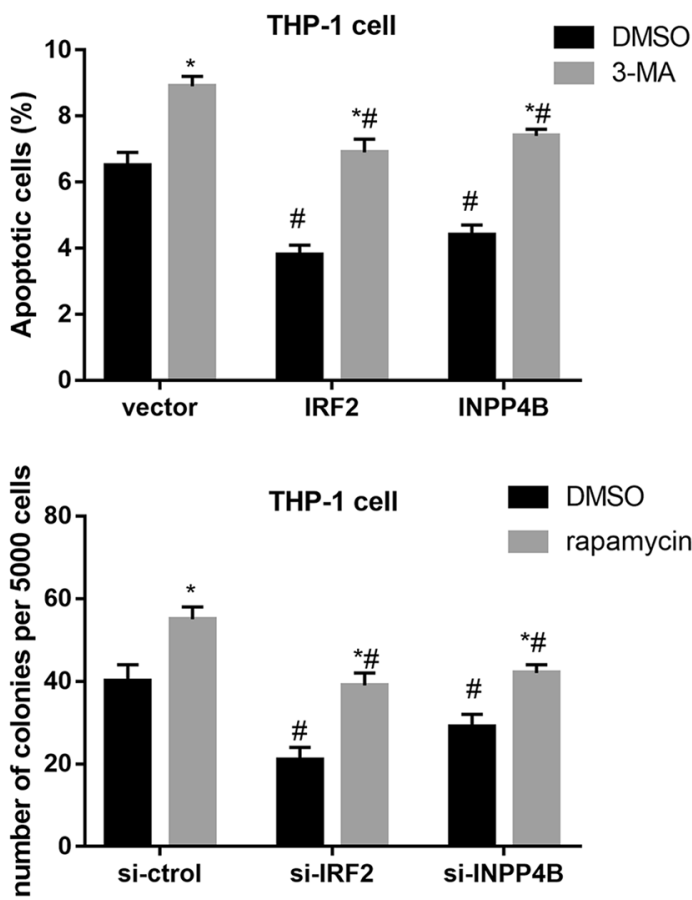

THP-1 cell

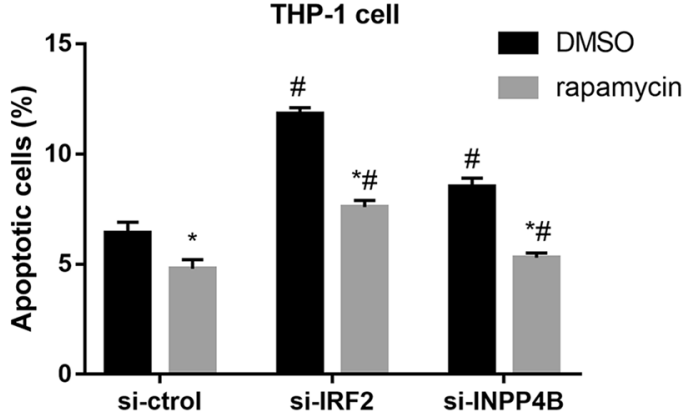

Fig. 5 Effect of IRF2-INPP4B on proliferation and apoptosis of AML cells. The cell proliferation (a) and apoptosis assays (b) in OCI/AML-2 and THP-1 cells transfected with vector, IRF2 and INPP4B after treatment with 3-MA. The cell proliferation (c) and apoptosis assays (d) in OCI/AML-2 and THP-1 cells transfected with vector, IRF2 and INPP4B after treatment with si-Ctrl, si-IRF2 and si-INPP4B. ${ }^{*} \mathrm{P}<0.05$ vs. the DMSO-treatment cells; ${ }^{\# P}<0.05$ vs. the si-Ctrl transfected cells 
Compelling evidence has delineated the carcinogenesis of INPP4B in breast cancer [21], laryngeal cancer [22] and melanoma [23]. Recently, accumulating evidence has strongly implied that INPP4B served as independent prognostic marker and were associated with colony formation, proliferation and chemotherapy resistance in AML patients [24-26]. The previous research has elucidated that IRF2 elevated the activity of INPP4B promoter by directly binding to its promoter to increase INPP4B expression in AML cells [13]. There was a positive correlation between INPP4B and Beclin-1 as well as LC3 mRNA expressions in AML cell lines. Besides, the overexpression of INPP4B promoted cell autophagy and proliferation and reduced cell apoptosis in AML cells, while lower expression level of INPP4B by si-INPP4B significantly promoted cell apoptosis and suppressed autophagy. Ko et al. found that autophagy inducer rapamycin restricted a feedback loop of NLRP3 inflammasome-p38 MAPK-NF- $k$ B pathways in autophagy- and p62-dependent manners [27]. In addition, previous studies uncovered that rapamycin, as a mTOR kinase inhibitor involved multiple signaling pathways such as Ras/ MEK/ERK, MAPK, JAK/STAT and Notch-1 pathways $[28,29]$. Thus, in the present study, the silencing of IRF2 or INPP4B could partially but not completely reverse the apoptosis-promoting effect of rapamycin.

\section{Conclusions}

In summary, we provided the first demonstration that IRF2-INPP4B axis inhibited the apoptosis of AML cells via inducing autophagy in vitro, and thus may be a new target for gene therapy in AML.

\section{Abbreviations \\ 3-MA: 3-methyladenine; AFP: alpha-fetoprotein; AML: acute myeloid leukemia; Atg5: autophagy-related gene 5; CRC: colorectal cancer; FBS: fetal bovine serum; GC: gastric cancer; GFP: green fluorescent protein; HCC: hepatocellular carcinoma; IRF: interferon regulatory factor; IRF2: interferon-regulatory factor 2; INPP4B: inositol polyphosphate-4-phosphatase, type-II; LC3: light chain 3; NSCLC: non small cell lung cancer; OS: overall survival; PCNA: proliferating cell nuclear antigen; QRT-PCR: quantitative real time PCR; siRNA: small interference RNA; TNM: tumor-node-metastasis. \\ Authors' contributions \\ FZ, PZ conceived and designed the experiments. FZ, JL, JZ, LL, KZ, PZ performed the experiments. FZ, SC, RL, PZ analyzed the data. FZ contributed reagents/materials/analysis tools. PZ wrote the draft paper. All authors read and approved the final manuscript.}

\section{Author details}

${ }^{1}$ Department of Hematology, The First Affiliated Hospital of Bengbu Medical College, No. 287 Changhuai Road, Bengbu 233004, Anhui, People's Republic of China. ${ }^{2}$ Department of Hematology, Bengbu Medical College, No. 287 Changhuai Road, Bengbu 233004, Anhui, People's Republic of China. ${ }^{3}$ Department of Electrocardiogram, The First Affiliated Hospital of Bengbu Medical College, No. 287 Changhuai Road, Bengbu 233004, Anhui, People's Republic of China.
Acknowledgements

Not applicable.

\section{Competing interests}

The authors declare that they have no competing interests.

\section{Availability of data and materials}

The datasets used and/or analysed during the current study are available from the corresponding author on reasonable request.

\section{Consent for publication \\ Not applicable.}

Ethics approval and consent to participate

Not applicable.

\section{Funding}

This study was supported by the Anhui University Natural Science Research Project (KJ2018A0235) and Key Project of Natural Science Foundation of Bengbu Medical College (BYKY1743ZD).

\section{Publisher's Note}

Springer Nature remains neutral with regard to jurisdictional claims in published maps and institutional affiliations.

Received: 24 December 2018 Accepted: 22 February 2019

Published online: 15 March 2019

\section{References}

1. Papaemmanuil E, Gerstung M, Bullinger L, Gaidzik VI, Paschka P, Roberts ND, Potter NE, Heuser M, Thol F, Bolli N. Genomic classification and prognosis in acute myeloid leukemia. N Engl J Med. 2016;374(23):2209-21.

2. Dombret H, Gardin C. An update of current treatments for adult acute myeloid leukemia. Blood. 2016;127(1):53-61.

3. Moloudizargari M, Asghari MH, Ghobadi E, Fallah M, Rasouli S, Abdollahi M. Autophagy, its mechanisms and regulation: implications in neurodegenerative diseases. Ageing Res Rev. 2017;40:64-74.

4. Deretic V, Klionsky DJ. Autophagy and inflammation: a special review issue. Autophagy. 2018;14(2):1-4.

5. Tooze SA, Yoshimori T. The origin of the autophagosomal membrane. Nat Cell Biol. 2010;12(9):831-5.

6. Hasanpourghadi M, Majid NA, Mustafa MR. Activation of autophagy by stress-activated signals as a cellular self-defense mechanism against the cytotoxic effects of MBIC in human breast cancer cells in vitro. Biochem Pharmacol. 2018;152:174-86.

7. Song S, Tan J, Miao Y, Li M, Zhang Q. Crosstalk of autophagy and apoptosis: involvement of the dual role of autophagy under ER stress. J Cell Physiol. 2017;232(11):2977.

8. Yang J, Moon HG, Chettimada S, Jin Y. Cross-talk between apoptosis and autophagy in lung epithelial cell death. J Biochem Pharmacol Res. 2014;2(2):99.

9. Qiang L, Chen L, Atkinson JM, Claxton DF, Wang HG. Atg5-dependent autophagy contributes to the development of acute myeloid leukemia in an MLL-AF9-driven mouse model. Cell Death Dis. 2016;7(9):e2361 .

10. Watson AS, Riffelmacher T, Stranks A, Williams O, De Boer J, Cain K, MacFarlane M, McGouran J, Kessler B, Khandwala S, et al. Autophagy limits proliferation and glycolytic metabolism in acute myeloid leukemia. Cell Death Discov. 2015;1:15008.

11. Folkerts H, Hilgendorf S, Wierenga ATJ, Jaques J, Mulder AB, Coffer PJ, Schuringa JJ, Vellenga E. Inhibition of autophagy as a treatment strategy for p53 wild-type acute myeloid leukemia. Cell Death Dis. 2017;8(7):e2927.

12. Manzella L, Tirrò E, Pennisi MS, Massimino M, Stella S, Romano C, Vitale $\mathrm{SR}$, Vigneri P. Roles of interferon regulatory factors in chronic myeloid leukemia. Curr Cancer Drug Targets. 2016;16(7):594-605.

13. Zhang F, Zhu J, Li J, Zhu F, Zhang P. IRF2-INPP4B axis participates in the development of acute myeloid leukemia by regulating cell growth and survival. Gene. 2017;627:9. 
14. Li YR, Wen LQ, Wang Y, Zhou TC, Ma N, Hou ZH, Jiang ZP. MicroRNA-520C enhances cell proliferation, migration, and invasion by suppressing IRF2 in gastric cancer. FEBS Open Bio. 2016;6(12):1257-66.

15. Yi Y, Wu H, Gao Q, He HW, Li YW. Interferon regulatory factor (IRF)-1 and IRF-2 are associated with; prognosis and tumor invasion in HCC. Ann Surg Oncol. 2013;20(1):267-76.

16. Sakai T, Mashima H, Yamada Y, Goto T, Sato W, Dohmen T, Kamada K, Yoshioka M, Uchinami H, Yamamoto Y. The roles of interferon regulatory factors 1 and 2 in the progression of human pancreatic cancer. Pancreas. 2014;43(6):909-16.

17. Mei Z, Wang G, Liang Z, Cui A, Xu A, Yun L, Liu C, Yang Y, Long C. Prognostic value of IRF-2 expression in colorectal cancer. Oncotarget. 2017:8(24):38969.

18. Choo A, Palladinetti P, Holmes T, Basu S, Shen S, Lock RB, O'Brien TA, Symonds G, Dolnikov A. siRNA targeting the IRF2 transcription factor inhibits leukaemic cell growth. Int J Oncol. 2008;33(1):175-83.

19. Wang $X$, Zhang $X$, Wang G, Wang L, Lin Y, Sun F. Hsa-miR-513b-5p suppresses cell proliferation and promotes P53 expression by targeting IRF2 in testicular embryonal carcinoma cells. Gene. 2017;626:344-53.

20. Jin JJ, Liu YH, Si JM, Ni R, Wang J. Overexpression of miR-1290 contributes to cell proliferation and invasion of non small cell lung cancer by targeting interferon regulatory factor 2. Int J Biochem Cell Biol. 2017;95(12):113-20.

21. Gasser JA, Inuzuka H, Lau AW, Wei W, Beroukhim R, Toker A. SGK3 mediates INPP4B-dependent PI3K signaling in breast cancer. Mol Cell. 2014;56(4):595-607

22. Min JW, Kim Kl, Kim HA, Kim EK, Noh WC, Jeon HB, Cho DH, Oh JS, Park IC, Hwang SG. INPP4B-mediated tumor resistance is associated with modulation of glucose metabolism via hexokinase 2 regulation in laryngeal cancer cells. Biochem Biophys Res Commun. 2013;440(1):137-42.
23. Chen L, Cao Y, Rong D, Wang Y, Cao Y. MicroRNA-605 functions as a tumor suppressor by targeting INPP4B in melanoma. Oncol Rep. 2017:38(2):1276-86

24. Dzneladze I, He R, Woolley JF, Son MH, Sharobim MH, Greenberg SA, Gabra M, Langlois C, Rashid A, Hakem A. INPP4B overexpression is associated with poor clinical outcome and therapy resistance in acute myeloid leukemia. Leukemia. 2015;29(7):1485-95.

25. Rijal S, Fleming S, Cummings N, Rynkiewicz NK, Ooms LM, Nguyen NY, Teh TC, Avery S, Mcmanus JF, Papenfuss AT. Inositol polyphosphate 4-phosphatase II (INPP4B) is associated with chemoresistance and poor outcome in AML. Blood. 2015;125(18):2815-24.

26. Wang P, Ma D, Wang J, Fang Q, Gao R, Wu W, Cao L, Hu X, Zhao J, Li Y. INPP4B-mediated DNA repair pathway confers resistance to chemotherapy in acute myeloid leukemia. Tumor Biol. 2016;37(9):1-11.

27. Ko JH, Yoon SO, Lee HJ, Oh JY. Rapamycin regulates macrophage activation by inhibiting NLRP3 inflammasome-p38 MAPK-NFkappaB pathways in autophagy- and p62-dependent manners. Oncotarget. 2017;8(25):40817-31.

28. Butler DE, Marlein C, Walker HF, Frame FM, Mann VM, Simms MS, Davies BR, Collins AT, Maitland NJ. Inhibition of the PI3K/AKT/mTOR pathway activates autophagy and compensatory Ras/Raf/MEK/ERK signalling in prostate cancer. Oncotarget. 2017;8(34):56698-713.

29. El-Habr EA, Levidou G, Trigka EA, Sakalidou J, Piperi C, Chatziandreou I, Spyropoulou A, Soldatos R, Tomara G, Petraki K, et al. Complex interac tions between the components of the PI3K/AKT/mTOR pathway, and with components of MAPK, JAK/STAT and Notch-1 pathways, indicate their involvement in meningioma development. Virchows Arch. 2014;465(4):473-85.
Ready to submit your research? Choose BMC and benefit from:

- fast, convenient online submission

- thorough peer review by experienced researchers in your field

- rapid publication on acceptance

- support for research data, including large and complex data types

- gold Open Access which fosters wider collaboration and increased citations

- maximum visibility for your research: over 100M website views per year

At BMC, research is always in progress.

Learn more biomedcentral.com/submissions 